\title{
Optimized Lieb-Oxford bound for the exchange-correlation energy
}

\author{
Garnet Kin-Lic Chan and Nicholas C. Handy \\ Department of Chemistry, Lensfield Road, Cambridge CB2 1EW, United Kingdom
}

(Received 14 September 1998)

\begin{abstract}
Using the ideas of Lieb and Oxford [Int. J. Quantum Chem. 19, 427 (1981)], we show that the exchangecorrelation energy, and indirect part of the Coulomb energy, are bounded from below by $-1.6358 \int \rho^{4 / 3}(x) d x$, where $\rho(x)$ is the single-particle density. [S1050-2947(99)02003-X]

PACS number(s): 31.15.Ew, 71.15.Mb
\end{abstract}

In recent years, Kohn-Sham density-functional theory $[1,2]$ has become the dominant computational method in many-body physics. In its usual formulation, we are concerned only with antisymmetric wave functions $\Psi$, and define functionals in terms of the single-particle density $\rho$, namely, the total energy $E[\rho]$, noninteracting kinetic energy $T_{s}[\rho]$, direct Coulomb repulsion $J[\rho]$, and exchangecorrelation energy $E_{x c}[\rho]$, through

$$
\begin{aligned}
E[\rho] & =\min _{\Psi \rightarrow \rho}\langle\Psi|\hat{T}+\hat{V}| \Psi\rangle+\int \rho(x) v(x) d x \\
& =T_{s}[\rho]+J[\rho]+E_{x c}[\rho]+\int \rho(x) v(x) d x, \\
T_{s}[\rho] & =\min _{\Psi \rightarrow \rho}\langle\Psi|\hat{T}| \Psi\rangle, \\
J[\rho]= & \frac{1}{2} \iint \frac{\rho(x) \rho(y)}{|x-y|} d x d y,
\end{aligned}
$$

where $v(x)$ is the external potential, $\hat{T}=-\Sigma_{i} \frac{1}{2} \nabla_{i}^{2}, \hat{V}$ $=\sum_{i<j}\left|x_{i}-x_{j}\right|^{-1}, x, y \in \mathbb{R}^{3}$, and here and throughout this work, we use Hartree atomic units. All correlation effects are incorporated into the exchange-correlation functional $E_{x c}[\rho]$, whose form is unfortunately unknown.

To make progress in density-functional theory, better exchange-correlation functionals are required. For example, current functionals have severe difficulties in predicting reaction barrier heights [3], or long-range correlations between molecules [4]. An important technique in developing new functionals is to construct expressions that obey rigorous mathematical bounds. Many such bounds have been proposed [5]. A particularly beautiful result was obtained by Lieb and Oxford [6], who proved that the indirect part of the Coulomb energy $I_{\Psi}=\langle\Psi|\hat{V}| \Psi\rangle-J[\rho]$ is bounded from below, viz.,

$$
I_{\Psi} \geqslant-C_{L} \int \rho^{4 / 3}(x) d x,
$$

where $C_{L} \leqslant 1.68$. However, the bound is not quite in the form we require for density-functional theory. For this, we must relate $I_{\Psi}$ and $E_{x c}[\rho]$, by choosing $\Psi$ to be $\Psi_{m}$, the minimizing wave function in Eq. (1), giving

$$
I_{\Psi_{m}}=E_{x c}[\rho]-\left(\left\langle\Psi_{m}|\hat{T}| \Psi_{m}\right\rangle-T_{s}[\rho]\right),
$$

where the quantity in brackets, the correlation kinetic energy, is positive semidefinite [since $T_{s}[\rho]$ is defined through the minimization in Eq. (3)]. This gives the density-functional formulation of the Lieb-Oxford bound as

$$
E_{x c}[\rho] \geqslant-C \int \rho^{4 / 3}(x) d x
$$

This introduces a new constant $C$, which is the constant of interest in density-functional theory, and from equality (6), satisfies $C \leqslant C_{L}$. Using additional data from the low-density limit of jellium, Perdew found a lower bound for $C$, giving $1.43 \leqslant C \leqslant 1.68[7]$.

The power of the Lieb-Oxford bound in densityfunctional theory lies in the fact that it is a direct bound on the energy (rather than the scaling behavior of $E_{x c}[\rho]$, say) and is simply a local integral of the density, in the form of the Dirac exchange functional for a plane-wave determinant [8]. To our knowledge, it is the only constraint on $E_{x c}[\rho]$ of this form. To illustrate its applicability, we consider the generalized gradient approximation (GGA), $E_{x c}^{\mathrm{GGA}}$ functionals, which are written as

$$
E_{x c}^{\mathrm{GGA}}[\rho]=-\kappa \int \rho^{4 / 3}(x) F_{x c}[\rho, \zeta] d x,
$$

where $\kappa=0.7386$, and $\zeta=|\nabla \rho| / \rho^{4 / 3}$. The flavor of GGA functional is determined by $F_{x c}[\rho, \zeta]$, which is an enhancement factor that corrects the Dirac exchange functional. We see that the Lieb-Oxford bound imposes limits on acceptable $F_{x c}[\rho, \zeta]$, viz., for all $\rho, \zeta$,

$$
F_{x c}(\rho, \zeta) \leqslant C / \kappa
$$

Using the value of $C$ given by Lieb and Oxford, we find $F_{x c}(\rho, \zeta) \leqslant 2.27$.

From the above, it is of some interest to densityfunctional development to have the tightest form of the LiebOxford bound. This is the question we address in this paper. The lower bound of Perdew suggests that the best value of $C$ in Eq. (7) requires only a small improvement in the LiebOxford proof. In fact, by refining the original proof, we show that the best value of $C$ or $C_{L}$ is less than or equal to 1.6358 , i.e., $I_{\Psi} \geqslant E_{x c}[\rho] \geqslant-1.6358 \int \rho^{4 / 3}(x) d x$. This improvement is 
significant, as it lowers the uncertainty in the optimum $C$ by almost $20 \%$, and is of the order of the correlation energy, which cannot be neglected.

Our method addresses a small incompleteness in the original work of Lieb and Oxford. However, for this paper, the proof is too long to repeat, and many of the details are not required. Instead we briefly sketch the proof, presenting only the relevant steps, and the reader is referred to the original paper for further details.

The Lieb-Oxford proof relies on a chain of three inequalities to bound $I_{\Psi}$. For this work, the most important is the first inequality, which bounds the operator $\hat{V}$. Earlier work of Onsager [9] and Lieb [10], showed that a bound for $\hat{V}$ may be obtained if we replace the point charges in $\hat{V}$ by hard spheres. This is because a bound for $\hat{V}$ cannot be separated from the problem of closest approach of charges. Lieb and Oxford used hard spheres with a density-dependent radius, and a charge distribution inside the spheres generated by scaling a function $\mu$, satisfying the following: (i) $\mu \geqslant 0$, (ii) $\mu$ is spherically symmetric around the origin and $\mu(x)=0$ if $|x|>1$, (iii) $\int \mu(x) d x=1$. This leads to the bound $\widetilde{V} \leqslant \hat{V}$, where $\widetilde{V}$ involves double integrals of the form $D[f, g]$ $=\frac{1}{2} \iint f(x) g(y)|x-y|^{-1}$, where $f, g$ are combinations of $\mu(x)$ and $\rho(x)$.

Next, we can bound the total Coulomb energy from above by $\langle\Psi|\widetilde{V}| \Psi\rangle$. Carrying out the integrations, and rearranging the resulting expression to subtract the direct Coulomb repulsion, leads directly to a bound for $I_{\Psi}$ involving the double integrals $D[f, g]$. However, it is not yet in the form (7). One reduces this bound by applying Hölder's inequality, and an inequality involving cutoffs in the kernel of the double integrals. This yields the bound for $I_{\Psi}$ in the form (7), where the constant $C_{L}$ is in terms of the charge distribution function $\mu$, viz.,

$$
I_{\Psi} \geqslant-\frac{3}{2}\left(6 K[\mu] D[\mu, \mu]^{2}\right)^{1 / 3} \int \rho^{4 / 3}(x) d x .
$$

$K[\mu]$ is a functional of $\mu$, namely,

$$
\begin{aligned}
K[\mu] & =\int_{0}^{1}\left[\frac{\partial}{\partial a} F(a,|x|)_{a=1}\right]_{+} d x, \\
F(a, r) & =a x^{-1}-a^{4 / 3} \phi\left(a^{1 / 3} x\right),
\end{aligned}
$$

where in Eq. (11) the + notation indicates cutoff, i.e., $h_{+}$ $=h$ if $h \geqslant 0, h_{+}=0$ if $h \leqslant 0$, and in Eq. (12), $\phi$ is the Coulomb potential generated by $\mu(x)$.

So far the constant $\left.C_{L}=-\frac{3}{2}(6 K[\mu] D[\mu, \mu]]^{2}\right)^{1 / 3}$ is not completely determined, for one needs to optimize the charge distribution function $\mu$. Lieb and Oxford proceed by saying " . . a a variational argument shows that the optimum choice of $\mu$ would the uniform ball if $\left[(\partial / \partial a) F(a,|z|)_{a=1}\right]_{+}$were replaced by $(\partial / \partial a) F(a,|z|) \quad \ldots$ however, trial and error indicates that this is approximately best with the cutoff.',

The idea in this paper is simple. The largest part of the work in obtaining a bound for $I_{\Psi}$ and $E_{x c}[\rho]$ has already been done in the construction used to arrive at Eq. (10). Moreover the construction is sound, i.e., only three inequalities are used to bound $I_{\Psi}$ (four for $E_{x c}[\rho]$ ), and they are all
TABLE I. Optimized coefficients $a_{i}$ for $\mu$.

\begin{tabular}{cccccc}
\hline \hline$a_{0}$ & $a_{1}$ & $a_{2}$ & $a_{3}$ & $a_{4}$ & $a_{5}$ \\
\hline-2.1486 & 12.8945 & 3.5500 & -63.9959 & 71.3860 & -21.8137 \\
\hline \hline
\end{tabular}

quite tight. Thus we feel it is best to finish things properly rather than by "trial and error," to find the exact optimum $\mu$ in Eq. (10). It follows that a better optimized $\mu$ leads to a better $C_{L}$, and thus also $C$.

Since $K[\mu]$ involves a cutoff in the kernel, it is easiest to approach the optimization problem numerically. To enforce the normalization and positivity constraints (i) and (iii) on $\mu$, we write

$$
\mu(x)=\frac{\alpha^{2}(x)}{\int \alpha^{2}(x) d x},
$$

with $x \in[0,1]$. Next, we model $\alpha(x)$ in two ways, with $n$ parameters, as a polynomial of order $n-1$,

$$
\alpha(x)=a_{0}+a_{1} x+\cdots a_{n-1} x^{n-1},
$$

or by a finite element model, i.e., $n$ values $a_{i}$ at evenly spaced points $x_{i}$, interjoined with straight line segments,

$$
\alpha(x)=\frac{a_{i+1}-a_{i}}{n-1}\left(x-x_{i}\right)+a_{i}, \quad x_{i} \leqslant x \leqslant x_{i+1} .
$$

By minimizing the prefactor $C_{L}$ with respect to the coefficients $a_{i}$, we obtain the optimum $\mu$.

In our calculations, all integrals were performed analytically except for the $x$ integration in $K[\mu]$ [see Eq. (11)], where standard Romberg integration [11] (accurate to 1 in $10^{8}$ ) was used. The minimization was carried out with two algorithms: multistart minimizations from randomly generated points (between 100-200) using the Nelder-Mead downhill simplex, and conjugate gradient search [11], with a convergence criterion of $5 \times 10^{-6}$ in the function value.

Using six parameters, we found an optimum $\mu$, which gave the upper bound $C_{L} \leqslant 1.6407$ with $K=0.32216$, $D=0.82284$. Values of $a_{0}, \ldots, a_{5}$ for the polynomial model (14), corresponding to this value of $C_{L}$, are given in Table I (solid line), and the corresponding $\mu$ is plotted in Fig. 1. The optimum value of $C_{L}$ did not change appreciably, even when we increased the number of parameters $n$ to 101 , where we found a best value of $C_{L} \leqslant 1.6358$ with $K=0.37313$, $D=0.76301$. It is hard to analyze the structure of $\mu$, but we note that the "large bump" exhibited by the polynomial approximation (the solid line in Fig. 1), between $x=0.2$ and $x=0.7$, is also a feature of the finite element model (the dashed line in Fig. 1). As can be seen, the finite element model exhibits considerable noise.

Also, due to the flatness of the $a_{i}$ surface, many different choices of $a_{i}$ yield similar $C_{L}$. We attempted to optimize using the downhill simplex simulated annealing algorithm [11], but did not find a lower value of $C_{L}$. For these reasons, we believe that, within the framework of the original LiebOxford proof, the upper bound of $C_{L} \leqslant 1.6358$ is optimized 


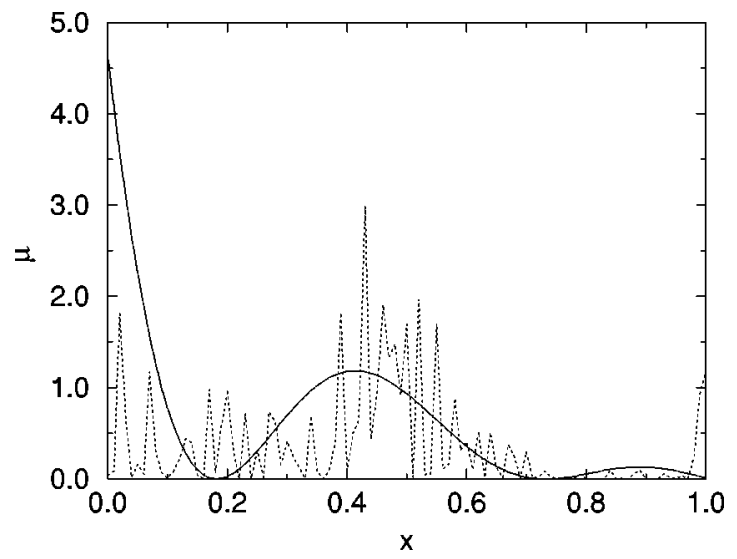

FIG. 1. An optimized charge distribution $\mu$. Quantities are in a.u. Solid line, six-parameter polynomial; dashed line, 101parameter finite element.

to approximately four significant figures. It follows, since $C_{L} \geqslant C$, that we have also determined an optimized $C$ $\leqslant 1.6358$.

How good is our improved Lieb-Oxford bound? Levy and Perdew conjectured that the optimum $C$ in Eq. (7) is just the lower bound of 1.43 [12]. Our result does not contradict this, since we have optimized $C_{L}$, and $C \leqslant C_{L}$. However, since the correlation kinetic energy $\left\langle\Psi_{m}|\hat{T}| \Psi_{m}\right\rangle-T_{s}[\rho]$ almost certainly does not vanish at the extreme $\Psi_{m}$ and $\Psi$ that yield equalities in Eqs. (5) and (7), and typical values of the correlation kinetic energy of the order of $10 \%$ of $E_{x c}[\rho]$, we conjecture that the optimum $C_{L}$ and $C$ differ by $\sim 0.1$. It, therefore, seems likely that one should not hope to lower our value of $C_{L}$ by very much. There may still be some room for improvement over our value of $C$. However, this will require further insight into the difficult correlation kinetic energy term in Eq. (6).

Finally, we finish with a brief test of our optimized LiebOxford bound. We have $C \leqslant 1.6358$, which in the context of the GGA [see Eq. (8)] requires the enhancement factor to satisfy

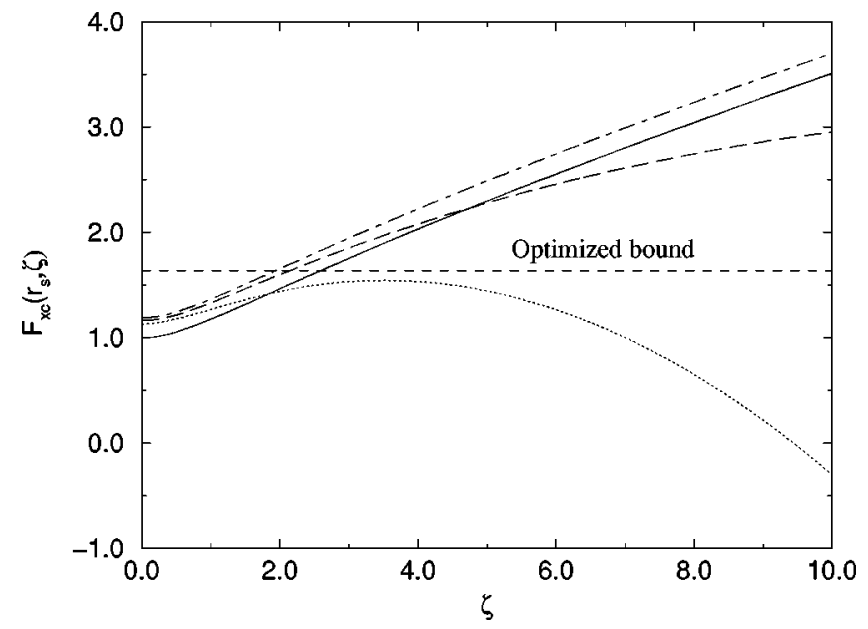

FIG. 2. Plot of the enhancement factor $F_{x c}\left(r_{s}, \zeta\right)$ for the BLYP functional. Quantities are in a.u. Dotted-dash, $r_{s}=\infty$; solid, $r_{s}$ $=10$; long dash, $r_{s}=3$; dotted, $r_{s}=0$.

$$
F_{x c}(\rho, \zeta) \leqslant 2.2146 \text {. }
$$

For the exact $E_{x c}[\rho]$, which also satisfies

$$
F_{x c}(\rho, \zeta) \geqslant F_{x c}\left(\rho^{\prime}, \zeta\right), \quad \rho>\rho^{\prime},
$$

it follows that bound (16) is most tight in the low density limit $\rho \rightarrow 0$.

Bound (16) is nontrivial. In Fig. 2 are some plots of $F_{x c}\left(r_{s}, \zeta\right)$, where $r_{s}=(4 \pi \rho / 3)^{1 / 3}$, using the common exchange-correlation functional of [13] (BLYP). We see that it clearly violates Eq. (16). Other than the local-density approximation [14], we know of only two GGA functionals that satisfy Eq. (16) (PW91), Ref. [15], which was constructed explicitly such that $F_{x c}(\rho, \zeta) \leqslant 1.93$ and PBE (Ref. [16]) which was constructed such that $F_{x c}(\rho, \zeta) \leqslant 2.273$. We advocate the use of our optimized bound as an updated constraint for new functionals.

G.K.-L.C. acknowledges Subrown Daitou and Romanos Daniel for useful discussions, and Aron J. Cohen for helping us produce Fig. 2.
[1] W. Kohn and L. J. Sham, Phys. Rev. 140, A1133 (1965).

[2] R. G. Parr and W. Yang, Density-Functional Theory of Atoms and Molecules, 2nd ed. (Oxford University Press, New York, 1989).

[3] F. A. Hamprecht, A. J. Cohen, D. J. Tozer, and N. C. Handy, J. Chem. Phys. 109, 6264 (1998).

[4] J. M. Pérez-Jordá and A. D. Becke, Chem. Phys. Lett. 233, 134 (1995)

[5] J. P. Perdew and K. Burke, Int. J. Quantum Chem. 57, 309 (1996)

[6] E. H. Lieb and S. Oxford, Int. J. Quantum Chem. 19, 427 (1981).

[7] J. P. Perdew, in Electronic Structure of Solids '91, edited by P. Ziesche and H. Eschrig (Akademie Verlag, Berlin, 1991).

[8] P. A. M. Dirac, Proc. Cambridge Philos. Soc. 26, 376 (1930)
[9] L. Onsager, J. Phys. Chem. 43, 189 (1939).

[10] E. H. Lieb, Phys. Lett. 70A, 444 (1979).

[11] W. H. Press, S. A. Teukolsky, W. T. Vetterling, and B. P. Flannery, Numerical Recipes in Fortran, The Art of Scientific Computing, 2nd ed. (Cambridge University Press, Cambridge, 1992).

[12] M. Levy and J. P. Perdew, Phys. Rev. B 48, 11638 (1993).

[13] A. D. Becke, Phys. Rev. A 38, 3098 (1988); C. Lee, W. Yang, and R. G. Parr, Phys. Rev. B 37, 785 (1988).

[14] By local-density approximation we refer to the combination of the Dirac exchange, and Vosko-Wilk-Nusair correlation functionals, S. J. Vosko, L. Wilk, and M. Nusair, Can. J. Phys. 58, 1200 (1980).

[15] J. P. Perdew and Y. Wang, Phys. Rev. B 45, 13244 (1992).

[16] J. P. Perdew, K. Burke, and M. Ernzerhof, Phys. Rev. Lett. 77, 3865 (1996). 